\title{
Virtual Personas: A Case Study on Truck Cabin Design
}

\author{
Jos Thalen and Mascha van der Voort \\ University of Twente, Enschede 7500AE, The Netherlands \\ $\{j \cdot$ p.thalen, m.c.vandervoort $\}$ @utwente.nl \\ http://www.ctw. utwente.nl/opm/en
}

\begin{abstract}
User involvement can help designers reach beyond functionality and usability, and identify the user's deeper needs for a pleasurable product experience. In practice, direct user involvement can be limited by a lack of knowledge of appropriate techniques, confidentiality constraints or limited access to end-users. Alternatively, personas can be used as a substitute for direct user involvement. Personas, however, often end up as posters in the hallway of a design department without being used, for instance because personas are not sufficiently realistic, or because the personas are insufficiently communicated within the design department. This paper presents a case study featuring Virtual Personas. This application allows designers to create and review use scenarios in a virtual world, featuring digital avatars. Although the application has been successfully deployed, it was found that additional effort is required for designers to really reach beyond the level of functionality and usability.
\end{abstract}

Keywords: personas, scenarios, roleplaying, virtual reality, case study.

\section{Introduction}

Product design is no longer driven by functionality alone. In addition to usefulness and ease of use, experiential qualities such as pleasure of use are now also playing an important role [1. Consequently, the need to inform the design process about the expectations of the users, as early as possible, is vital. A study of the current state of user involvement in design practice, in which the authors were also involved, revealed however that design practice has not yet caught up with design literature [2]. The study captured the current state of user involvement in design practice, and identified common pitfalls and challenges faced in design practice, such as a lack of concrete user involvement techniques, a nonuser centred company culture and several practical constraints such as limited access to end-users and confidentiality issues.

This paper presents a case study carried out for one of the companies that was also involved in the aforementioned study, as part of a larger research project [3]. The company, situated in the Netherlands, is involved in the development, production, marketing and sale of medium and heavy-duty commercial vehicles. The company's design department faces several challenges with respect to user 
involvement. Firstly, direct user involvement is not possible because of confidentiality constraints. The designers therefore have to rely on input from the marketing department (which conducts interviews and surveys) and on the input from end-user representatives; company employees who have a truck drivers licence. Furthermore, the attempts to increase indirect user involvement through personas have failed because of a lack of concrete guidance on how to adopt them in the design process. The case study introduces Virtual Personas. With Virtual Personas, personas are represented by interactive digital avatars rather than text based descriptions or photos. The virtual personas 'live' in an interactive virtual environment, in which designers can act out future use scenarios, reflect on product proposals or generate and discuss new ideas.

The paper is structured as follows. In section 2 we describe the background of the techniques that form the basis of the Virtual Persona technique. Section 2.1 introduces personas and several related techniques, including scenario based design and roleplaying. Section 2.2 briefly outlines the current state of VR (Virtual Reality) techniques in the field of product design, particularly looking at VR applications in the early stages of the product design process. Section 3 presents the approach and proceedings of the case study, and presents an evaluation of the results. In section 4 the paper reflects on the case study and discusses the contributions to design practice.

\section{Background}

The following subsections introduce the traditional persona technique and further explain related design techniques, including scenario based design and role playing. Subsequently, a brief introduction to VR is presented along with a motivation for using this technique as a new medium for personas.

\subsection{Personas, Scenarios and Roleplay}

A definition of personas is given in [4]: User models, or personas, are fictional, detailed archetypical characters that represent distinct groupings of behaviours, goals and motivations observed and identified during the research phase. These user models should become a 'real' person in the mind of the designers, and help them to make appropriate design decisions 'on behalf' of that user. Since its introduction by [5] in the '90s, personas have been widely discussed in literature. In this overview however, we focus on the practical aspects of using personas.

In design practice, a common challenge of working with personas is the lack of concrete guidelines on how to actually apply them in a design process, as for instance discussed in [6. The authors describe additional challenges, such as a lack of believable personas, a lack of communication of the personas, and a lack of high-level support or encouragement. These issues are addressed by presenting an approach for creating believable personas, and by proposing concrete design techniques that use and communicate personas, such as priority matrices, posters and foundation documents. 
Another development that intends to make personas a more engaging design technique is the combination of personas with scenarios. Scenarios, as introduced in 7, are stories that describe the use of a product by a user, in a certain setting. In [8] it is proposed to use personas to make the 'user' (or actors) in scenarios more engaging. Seeing personas in a specific context makes it easier to understand their actions and consequently help move designers in 'the right direction'. In [9] it is argued that scenarios should be formed around personas to "[...] obtain a far more powerful level of identification and engagement [...]", similar to how people identify with characters from a soap opera series on TV. Similarly, in [10], theatre techniques were used to effectively communicate personas and scenarios within a group of software designers. While human role playing transfers personas from paper to more 'engaging' stories, there are some drawbacks. Firstly, it takes quite some time to get used to acting out scenarios in front of colleagues. Alternatively, professional actors could be used, but this may lead to an increase in time and costs. Secondly, role playing still takes quite some imagination to 'see' the correct context in which a story is taking place. Thirdly, analysing the data from such sessions is time consuming; raw video has to be analysed in order to extract information relevant for the project.

Combining personas, scenarios and role play is expected to contribute to the adoption of personas by the designers of the company involved in the case study. However, given the aforementioned drawbacks of traditional role playing, we propose to use VR technologies to create virtual environments in which designers can use virtual personas to create, act out and evaluate scenarios. The following section further discusses the opportunities of VR.

\section{$2.2 \quad$ Virtual Reality}

VR technologies create an alternative reality in which worlds, objects and characters can be experienced that may not yet be available in reality. VR was initially considered a high-end design technique for large industries such as aerospace and automotive design. Over the years however, advancements in hardware and software reduced costs and extended the application scope of VR to simulation, training, prototyping and evaluation purposes (see [11 for an extensive overview).

Given the increasing availability of off-the-shelf VR hardware and software, it is becoming an interesting technique to facilitate user involvement in the early stages of the design process. In [12] it is argued that successfully user involvement requires explicit representations of design information, realistic interaction between the user and design information, and users should become conscious of the consequences of design decisions. These requirements are in line with the characteristics of VR: it can provide realistic and interactive representations of (future) products, allowing end-users to create, experience and assess future use situations. 13 provides a more extensive overview of example applications.

In the current case study VR is employed to create engaging, realistic and interactive representations of use scenarios, featuring virtual personas. It is expected that, through this new medium, designers are encouraged to step in to 
the shoes of specific personas, and reach a deeper level of understanding of their needs, wishes and desires.

\section{Case Study}

The case study focuses on the design of the truck cabin, from which truck drivers not only drive the truck, but, especially in case of long distance trips, in which they live as well. The design of the cabin therefore has a significant effect on how the truck is experienced by the driver. This goes beyond the driving characteristics of the truck itself. It concerns the layout of the interior, the lighting design, in-vehicle media system user interfaces and the design of the sleeping area.

The vehicle definition department is in charge of cabin design. A separate product planning department provides this department with insights in current market needs, not only from vehicle drivers but also from vehicle buyers. The insights are gathered through interviews and field studies carried out by the product planning department. Apart from this indirect user involvement, user involvement is limited by confidentiality constraints. Consequently, in order for the vehicle definition department to conduct tests and evaluations they have to rely on end-user representatives rather than actual end-users. End-user representatives are co-workers who either have experience as a truck driver themselves, or are in direct contact with real end-users.

As explained in the introduction, the designers attempted to address the lack of direct end-user involvement by creating personas. Rather than working with statistical data from the product planning department, designers could use personas to think from the perspective specific individuals. However, a lack of concrete guidance on how to apply the personas in practice has caused the personas to fail in reaching designers and engineers.

Therefore, the problem definition of the case study is twofold. Firstly, the lack of direct end-user involvement in the design process makes it difficult for designers take future use scenarios and experiences into account. Secondly, a feasible solution to the first issue failed to be adopted in practice. The Virtual Persona application aims to provide a more vivid representation of the established personas. The application combines the advantages of role playing (resulting in engaging stories) with advantages of VR, namely the explicit and interactive visualisation of future use situations.

\subsection{Approach}

The case study addresses three research questions. Firstly, does the Virtual Persona session stimulate designers to put themselves in the shoes of end-users? If so, what kind of product insights (in terms of functionality, usability and experiential) can be obtained? Lastly, are there specific benefits of using VR techniques in this application? To investigate these questions, the Virtual Persona application will be deployed and evaluated using the following steps. 
1. Prototype development A functional prototype of the Virtual Persona application will be developed to allow the designers to experience what it is like to use the application in practice, and to provide the researcher with feedback on how to improve the application.

2. Test case A test case is carried out in which the application prototype is deployed. This phase also involves the definition of a design session structure of which the Virtual Persona application is part.

3. Evaluation The test case will be evaluated by transcribing and analysing video recordings of the session, and by gathering insights through postsession evaluation forms.

The following subsections further elaborate on the individual steps.

\subsection{Prototype Development}

A functional prototype of the envisioned Virtual Persona application was developed in collaboration with the participating designers from the company. The basis of the application resembles a theatre layout; a large screen is used to present a group of designers with a live view of the virtual world in which the scenarios are acted out. The virtual world includes an urban environment as well as a highway, a truck parking lot and a petrol station. The environment also includes interactive objects, such as autonomous traffic and driveable trucks. Within the virtual world, virtual personas are represented by digital avatars. The different types of personas are represented by their clothes, physical appearance and facial features (see figure 1). An on-screen user interface allows designers to control the avatars (e.g. their behaviour and movements in the virtual world), but also provides access to virtual world settings, such as lighting and camera viewpoints.

To facilitate rapid and flexible development of the prototype, the Blender Game Engine 1 was used as a development platform. This suite supports 3D modelling as well as behaviour modelling through scripts, real-time rendering and interfaces well with external devices such as the Microsoft Kinect. The latter allowed the researcher to experiment with using gestures to control the virtual personas. During an early review session however, designers did not see any added value in being able to control the avatars through gestures and motion tracking. The use of a mouse and keyboard controls was considered more effective; this would allow the designers to position the avatar anywhere in the virtual world by simply clicking on the desired position. Additionally, designers indicated that the virtual personas should be properly introduced before using them in a scenario. This could be achieved by creating a short introduction video of each persona. Furthermore, the visual appearance of the avatars should be improved by using more distinct clothing and facial textures, and by using different body models.

\footnotetext{
${ }^{1}$ See http://www.blender.org
} 

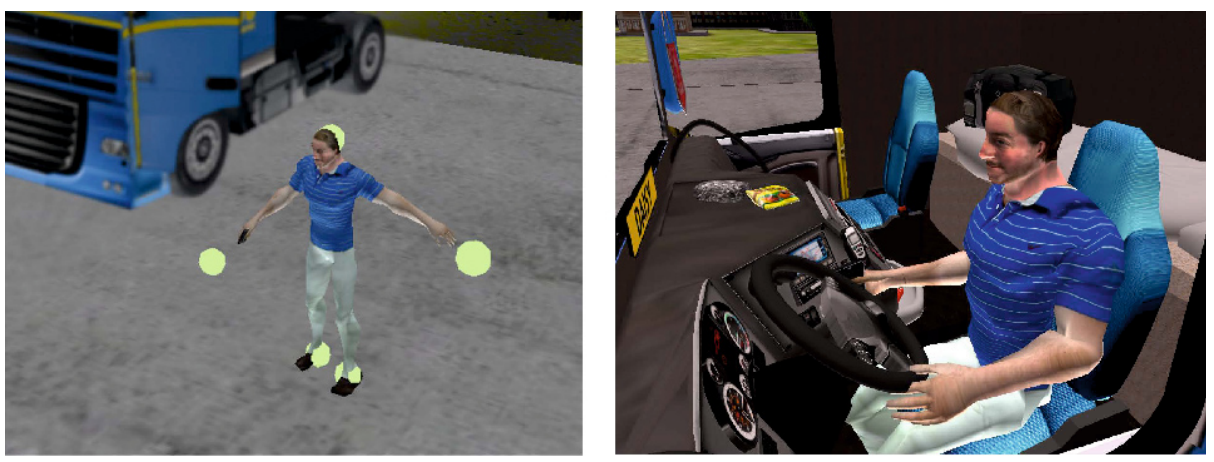

Fig. 1. Digital avatars represent specific personas. Mouse controls allow designers to control individual limbs using 'handles' and to automatically position personas, for instance on a seat in the cabin.

\subsection{Test Case}

In this step the prototype application is deployed in a realistic test case. The test case concerns the idea of developing a new device to control various truck functions, such as the lights and the central locking system. The feasibility of this idea is to be assessed by the participants in this session, who are experts in specific areas such as mechanical engineering or HMI design. In particular, the designers and engineers are to evaluate the concept from the perspective of two personas embodied in virtual avatars.

The test case comprises two identical one hour sessions, each involving four different participants from the company. Session participants include designers and engineers who will work on a fictional design case defined for this session. The two sessions were facilitated by the researcher, who made sure that the following steps were carried out.

1. Case Description (3 min.) - The participants are asked to assess a proposed product concept. This step introduces the product and explains the aim of the session.

2. Scenario Setting (2 min.) - A scenario is provided as a starting point for the brainstorm: You are near the end of a long driving day. Because of driving time regulations you are forced to spend the night at a trucker's parking space near the highway. It's around 17:00 when you arrive at the parking space. There is a gas station and small snack/restaurant nearby. After parking your truck, the evening starts...

3. Brainstorm (10 min.) - The participants are asked to brainstorm about what tasks a driver would have in this period of time and what events could take place. Participants write down the tasks and events on cards and put them on a whiteboard.

4. Persona Introduction (5 min.) - A short 1-minute video of each persona (Jim and Stanley) is shown. 'Jim' is a young and technology oriented driver, 


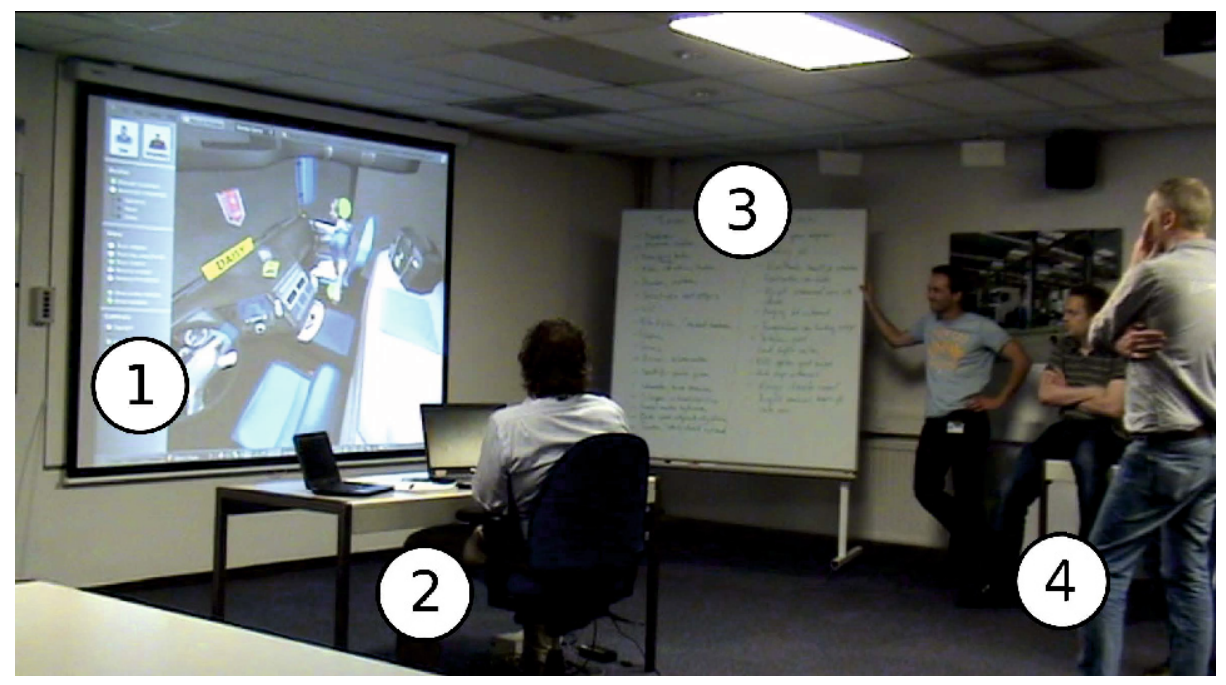

Fig. 2. An overview of the Virtual Persona session, involving the virtual environment (1), the operator (2), the tasks and events (3) and the design team (4)

who loves his job. 'Stanley' is an older driver, who drives his truck just to make money.

5. Scenario Generation (40 min.) - The task and event cards are used to create a scenario that can then be acted out in the virtual environment. The virtual environment is operated by one of the participants, supported where needed by the facilitator.

The proceedings of the test session are captured on video and the participants are asked to fill out an evaluation form after the session.

\subsection{Results}

This section briefly describes the proceedings of the test session, and summarises the insights gathered through the post-session evaluation forms. Section 4 further elaborates on the results.

Proceedings. Both groups successfully managed to complete the design session. During the sessions the virtual environment played a balanced role as one of four elements that interacted during the session (see figure 2). Throughout the session, there were interactions between the group of designers, the tool operator, the virtual environment and the list of tasks and events. In the first few minutes of each session designers systematically picked an item from the list of tasks and acted this out in the virtual environment. As the session advanced, most of the time was spent on group brainstorms and discussions, fed by information from the virtual environments or events from the list. 
Post-session Evaluation The post-session evaluation form consists of three parts, asking the participants to reflect on the persona engagement, the product insights and the added value of VR.

1. Persona Engagement. The majority of the participants (6 out of 8 ) indeed felt like they have been evaluating the product concept from two different user perspectives, and all participants consider this activity to be useful especially in the early stages of the PDP. Tables 1 and 2 list the expected benefits and drawbacks of using Virtual Personas. Furthermore, participants also mentioned some of the risks related to the use of (virtual) personas. For instance, it was noted that 'we [designers] should not presume to know everything about a user', and that the focus on personas sometimes distracted from the actual use case.

2. Product Insights. The insights gained during the session primarily include refined or new product ideas for the use case of the session 2 . The results of the evaluation indicate that participants expect 'Jim' to accept, like and use the application, while 'Stanley' will probably not use it. Furthermore, the consequences of not using the new product concept were discussed explicitly in Stanley's scenario, as illustrated by the following fragment:

"Stanley has to do the truck administration manually, from his driver seat. This takes a bit more time than doing it digitally, and it's not automatically updated with the home office. [...] He will give his wife a call to say he won't be eating at home. He uses his own phone for this."

This is also reflected by some of the answers to a question in which the designers are asked about the expected use of the product concept. In their answer, designers reason from the perspective of the persona: "Eventually yes, because it will make his job easier. The threshold is high, and he will only use a small part of all functions/features", and "Yes, he grew up with technology like this en is very capable of using them.".

3. Added Value of VR. The participants indicated that the virtual environment helps with imagining use situations and with explaining and understanding user-product relations. The environment sometimes reminds them of small details or very 'standard' tasks or actions that would otherwise have been overlooked. On the other hand, the use of the virtual environment sometimes distracted designers from a discussion. Furthermore, it lacks a good simulation of 'time pressure' (e.g. in real-life truck drivers are always trying to reach a destination in time, while the application did not include such aspects), and similar aspects may be forgotten if there are no 'triggers' present in the virtual environment. For instance, in this session objects like a coffee mug and a sandwich triggered designers to think about lunch, which may have been overlooked if the virtual sandwich and mug were not included in the environment. Lastly, the development and extension of the virtual environment is time consuming.

${ }^{2}$ The exact results of the session (i.e. the resulting scenarios, functions and requirements) are not included because of confidentiality reasons. 
Table 1. Potential benefits identified by designers after the test session

Potential benefits of using Virtual Personas

"Exchange experiences between participants"

"Seeing line of sight, imagining things, immersing in a situation"

"Interactions, and to remind you of simple things (such as opening doors)"

"Understanding/immersing in the user"

"Imagination/immersion in character"

"Especially in early stage it will help with setting boundary conditions and a scenario"

"You are encouraged to think about details"

"The tool keeps you focused, constantly reminding you of the persona"

Table 2. Potential drawbacks identified by designers after the test session

Potential drawbacks of using Virtual Personas

"Takes time to extend"

"Takes time and it may distract"

"Could be negative; you might forget things if the environment doesn't show them"

"Maybe we focused to much on the 'perfect user"

"Real circumstances are missing, such as time pressure. These make the experience slightly less realistic"

"It's time taking/cumbersome to work with the scenario, difficult to get the right level of detail"

"You can't do everything in the environment, it still takes some imagination"

\section{Discussion}

The Virtual Persona application succeeded in getting the designers to step into the shoes of two specific end-users. They acted out use situations, came up with new product functions, identified several problem scenarios and refined some of the preliminary product requirements. While this positively answers the first research question (see section 3.1) the remaining two questions should be further discussed.

\subsection{Product Insights}

As shown in section 3.4, the insights gained during the session primarily concern the functionality and usability of the product concept. Especially in the case of the tech-oriented 'Jim' persona, designers were constantly coming up with new functions for the product to provide. While idea generation is acceptable in the early stages of a design process, it can be questioned whether it is also desired in a review or evaluation session: the generation of new ideas distracts designers from looking into the deeper issues related to new product concepts or interaction styles.

Although the designers primarily came up with functional ideas, issues and requirements, there are indications that a deeper understanding of and engagement 
with the personas was achieved. While designers came up with new functions for 'Jim', they were also triggered to think about the consequences of these functions for 'Stanley'. Questions like 'will Stanley feel left out when we make a product like this?' were raised and discussed. For example, using a smartphone as a platform for various cabin functions would allow 'Jim' to easily fine tune his environment. 'Stanley', who does not use a smartphone, might feel like he is missing out on something. Participants started a discussion about what kind of cabin fine tuning would a person like Stanley need, or what kind of interactions should we design to accommodate Stanley's needs? These discussions illustrate that although starting on a superficial 'functional' level, the designers eventually touch the persona's deeper needs and requirements.

It is expected that, apart from the goal of the session, the persona descriptions and their level of detail have caused a focus on functionality and usability. The test case featured two relatively flat and extreme characters that easily evoke caricature behaviour: Jim prefers to have as much features in one product as possible, while Stanley is old fashioned and hates electronic devices. Similar to [5], it is expected that more detailed virtual personas will evoke more user specific product functions.

\subsection{Added Value of VR}

The results of the post-session evaluation forms indicate that participants find it difficult to pinpoint the added value of using a virtual environment and virtual personas. Although it is generally acknowledged that the explicit and interactive representation of personas in a future use situation is useful, it can be argued that this could also be achieved with live role playing, animated scenarios or storytelling. A formal comparison of virtual personas with such alternative techniques could provide more insight into the benefits or drawbacks. Based on the observations made during the test session however, there are several aspects considered to be unique to using virtual personas, and several areas of potential added value.

The primary benefit of using an interactive virtual environment is that even with a limited level of realism and only few means of real-time modification, it provides designers with a lot of freedom to explore foreseen as well as unforeseen use scenarios (created ad-hoc). In the test session we observed how small objects and actions can evoke new use scenarios. Even when a use scenario required the avatar to do something that was not pre-defined (e.g. checking tire pressure outside the cabin), an approximation of the actions and events was enough to initiate relevant discussions concerning this particular scenario. This level of flexibility is quite difficult to reach with pre-recorded animated scenarios or storyboards.

Another relevant benefit is that, compared to role playing or acting out scenarios, the Virtual Persona application is quite a safe way of stepping into the shoes of a persona. The designers, as a group, instruct the application operator how to act out the scenario. Especially in the field of engineering (e.g. truck manufacturing and machine design) this reduces the threshold for designers to 
express their ideas. Apart from the application operator, the participants do not need specific skills or preparation to contribute to the session.

Lastly, there are various potentially beneficial features that have not yet been implemented in the application prototype. For example, designers indicated that it would be useful to record the use scenarios generated during the sessions. The recordings can be used for future reference within the design team, but also to communicate the results to other departments. Furthermore, by recording the actual events in the 3D environment, it would be feasible to 'revisit' scenarios later on in the design process, and validate new product concepts in a specific use scenario. Another opportunity identified by the designers was for the environment to contain detailed $3 \mathrm{D}$ and $2 \mathrm{D}$ models. This would for instance allow designers to evaluate the user interface of a new dashboard or car radio from the perspective of personas, in specific use situations.

\section{Conclusion}

The Virtual Persona application helps designers to start thinking from the perspective of specific end-users. As such, it is a feasible alternative when direct end-user involvement is impossible. The results of the test session presented in section 3 show that designers mainly discussed product functionality, and to some extend usability and experience. As explained in section 4, it is expected that a deeper level of engagement with the virtual personas can be reached when more detailed persona descriptions are used. Additional factors include the time available for conducting a virtual persona session, and the number and diversity of virtual personas used.

The case study did not involve an explicit comparison of Virtual Personas to alternative techniques, such as animated scenarios or role playing. Nevertheless, we identified several unique aspects of this application, namely the level of flexibility designers have in creating and reviewing use scenarios and the low threshold for designers to contribute to the session. Additional opportunities, such as recording and re-visiting scenarios, have been proposed by the participating designers.

\subsection{Future Work}

We propose two directions of future work. Firstly, the application prototype and session structure developed in this case study can be used as a starting point for a more elaborate test case to further validate the effectiveness of the Virtual Persona application. In particular, a deeper level of insights (i.e. beyond functionality and usability) could be obtained when the shortcomings of the current test case, such as the limited time available for creating scenarios and the lack of sufficiently detailed and distinct personas, are addressed. Secondly, to gain insight in the added value of VR in the Virtual Persona application, the technical setup could be extended in two directions, namely a more high-end (e.g. using more detailed avatars, fully immersive VR and natural interfaces for controlling the avatars) and a low-end (e.g. using physical drawings or sketches). 
A comparison of these three versions will provide insight into whether VR actually adds something to traditional personas, and, if so, whether or not the current implementation is sufficient.

\section{References}

1. Jordan, P.W.: Designing Pleasurable Products: An Introduction to the New Human Factors. CRC Press (August 2002)

2. Ozcelik, D., Quevedo-Fernandez, J., Thalen, J., Terken, J.: Engaging users in the early phases of the design process: Attitudes, concerns and challenges from industrial practice. In: Proceedings of the 2011 Conference on Designing Pleasurable Products and Interfaces, DPPI 2011, pp. 13:1-13:8. ACM, New York (2011)

3. Thalen, J.: Facilitating user centred design through virtual reality. $\mathrm{PhD}$ thesis, University of Twente, Enschede. The Netherlands (2013)

4. Calde, S., Goodwin, K., Reimann, R.: SHS orcas: the first integrated information system for long-term healthcare facility management. In: Case Studies of the CHI 2002: AIGA Experience Design FORUM, CHI 2002, pp. 2-16. ACM, New York (2002)

5. Cooper, A.: The Inmates Are Running the Asylum. Macmillan Publishing Co., Inc., Indianapolis (1999)

6. Pruitt, J., Grudin, J.: Personas: practice and theory. In: Proceedings of the 2003 Conference on Designing for User Experiences, DUX 2003, pp. 1-15. ACM, New York (2003)

7. Carroll, J.M.: Scenario-based design: envisioning work and technology in system development. Wiley \& Sons, New York (1995)

8. Blomkvist, S.: The user as a personality. In: Position Paper for the Course Workshop on Theoretical Perspectives in Human-Computer Interaction at IPLab, KTH (2002)

9. Grudin, J., Pruitt, J.: Personas, participatory design and product development: An infrastructure for engagement. In: Proc. PDC, vol. 2002, p. 7 (2002)

10. Carmichael, A., Newell, A.F., Dickinson, A., Morgan, M.: Using theatre and film to represent user requirements. In: Proceeding of Include Conference Royal College of Art, London, vol. 5 (2005)

11. Jimeno, A., Puerta, A.: State of the art of the virtual reality applied to design and manufacturing processes. The International Journal of Advanced Manufacturing Technology 33(9-10), 866-874 (2006)

12. Tideman, M., van der Voort, M., van Houten, F.: A new product design method based on virtual reality, gaming and scenarios. International Journal on Interactive Design and Manufacturing 2(4), 195-205 (2008)

13. Thalen, J., van der Voort, M.: Facilitating user involvement in product design through virtual reality. In: Xinxing, T. (ed.) Virtual Reality - Human Computer Interaction, pp. 105-124. InTech (September 2012) 